\title{
Guggulu, une plante aux vertus hypolipémiantes pour nos sociétés rassasiées
}

\author{
Isabelle Dugail
}

$>$ La régulation de la concentration sanguine de cholestérol est un problème de plus en plus aigu dans nos sociétés pléthoriques, où l'hypercholestérolémie touche plus d'un individu sur 20. Au cours des dernières années, l'augmentation continue de la prévalence de l'obésité, à laquelle l'hypercholestérolémie est souvent associée, augure assez mal de l'amélioration de ce problème de santé publique. Compte tenu de cette situation, d'actives recherches sont en cours sur les mécanismes très complexes qui contrôlent l'homéostasie du cholestérol, et en particulier la concentration intracellulaire de cholestérol, élément vital pour le maintien de l'intégrité de la membrane plasmique et de ses capacités de signalisation.

À l'échelon de la cellule, le manque de cholestérol est détecté par un système qui n'est encore qu'incomplètement caractérisé. La mise en jeu de ce système conduit à l'activation des facteurs de transcription SREBP (sterol regulatory element binding protein) qui contrôlent, l'expression des enzymes responsables de la biosynthèse endogène du cholestérol et de son captage $(\rightarrow) \mathrm{m} / \mathrm{s}$ $1999, n^{\circ} 12$, p. 1472 connu comme l'étape initiale du transport inverse du cholestérol, s'effectue grâce à une protéine membranaire ABCAl (ATPbinding cassette), elle-même sous le contrôle de la concentration intracellulaire de cholestérol, et capable de transférer le cholestérol membranaire sur des accepteurs extracellulaires spécifiques, généralement des lipoprotéines de type HDL (high density lipoproteins). Le flux de cholestérol créé par ce transport inverse (communément appelé le «bon cholestérol ») aboutit au foie où il sera finalement éliminé par la voie biliaire.

Ainsi, on comprend comment la concentration en cholestérol circulant résulte de plusieurs paramètres : des apports exogènes via l'alimentation, de la capacité des cellules périphériques d'utiliser le cholestérol circulant, et de son élimination par la voie biliaire. Au cours des dernières années, la compréhension des mécanismes moléculaires qui contrôlent l'étape d'élimination biliaire a beaucoup progressé. En particulier, la régulation de l'expression de l'enzyme-clé de ce processus, la cholestérol $7 \alpha$ hydroxylase (CYP7Al) a été élucidée $(\rightarrow)$. Le facteur de transcription LXR, de la famille des exogène, ce qui permet à la cellule un apport continu et adapté en cholestérol. À l'inverse, toute accumulation excessive, qui pourrait avoir des conséquences néfastes parce qu'elle engendre la formation de cristaux insolubles, doit être évitée. Comme la plupart des cellules sont incapables de dégrader le cholestérol, l'excès est soit stocké sous une forme plus inoffensive (la forme estérifiée), soit éliminé dans la circulation. Ce processus,
Inserm U.465, 15, rue de

l'École de Médecine, 75270

Paris Cedex 06, France.

idugail@bhdc.jussieu.fr

Rebaptisé BAR (bile acid receptor) depuis que l'on sait que les acides biliaires sont les ligands (et donc les activateurs) physiologiques de FXR, ce facteur est l'élément charnière du rétro-contrôle négatif de la production d'acides biliaires. En effet, lorsque le pool d'acides biliaires augmente, l'activation de FXR conduit à l'arrêt de la transcription de CYP7Al. Le mécanisme supposé de cette inhibition transcriptionnelle fait intervenir un coinhibiteur SHP (short heterodimer partner). Comme SHP est une cible transcriptionnelle directe de FXR, et que SHP empêche l'activité transcriptionelle du dimère LXR/RXR, on comprend comment les acides biliaires inhibent par rétrocontrôle l'élimination biliaire du cholestérol. Toutes ces molécules peuvent être autant de cibles thérapeutiques potentielles pour combattre l'hypercholestérolémie. L'exemple des statines, cette classe d'inhibiteurs compétitifs de l'HMGCoA réductase, l'enzyme-clé de la biosynthèse endogène du cholestérol, qui regroupe des agents hypocholestérolémiants largement utilisés en clinique est à cet égard démonstratif, et médecine/ sciences s'est récemment fait l'écho d'une autre nouvelle molécule hypolipémiante décrite dans la littérature $(\rightarrow)$. Cette fois, c'est la revue Science [1] qui nous révèle une molécule originale ayant pour cible le facteur de transcription FXR. II s'agit de la gugglesterone (cis ou trans 4,17(20)-prégnadiène-3,16-dione), composé purifié à partir d'une résine (commiphora mukul) proche de la myrrhe, obtenue à partir $(\rightarrow) \mathrm{m} / \mathrm{s}$ 2002, n०6-7, p. 659 
d'un arbuste, qui pousse dans les régions arides de l'Inde. La gugglesterone tire son nom étrange de la plante dont elle est extraite, guggulu en sanscrit. Cette résine, connue depuis plus de 600 ans avant JC, était utilisée comme « purifiante » par la médecine traditionnelle indienne. Des articles parus à la fin des années 1980 dans des journaux scientifiques indiens [2, 3] avaient remis ses vertus au goût du jour, et rapporté ses propriétés hypocholestérolémiantes. Un groupe de chercheurs américains a élucidé le mécanisme par lequel cette molécule exotique réduit le cholestérol en démontrant que la gugglesterone est un antagoniste du facteur de transcription FXR, probablement capable, en inhibant son activité transcriptionnelle, de bloquer le rétro- contrôle négatif exercé par les acides biliaires sur la voie d'élimination du cholestérol.

Les auteurs démontrent dans des cellules transfectées, en utilisant des promoteurs artificiels inductibles par FXR, que cette molécule, qui, seule, n'a pas d'effet, inhibe l'activation transcriptionnelle de FRX par les acides biliaires. De même, dans des cellules intactes exprimant FXR, traitées par un acide biliaire (l'acide chénodéoxycholique), l'expression des gènes cibles de FXR, comme SHP, est inhibée par la gugglesterone. La notion selon laquelle la gugglesterone est un antagoniste de FXR repose sur plusieurs arguments expérimentaux : (1) dans des cellules transfectées avec des constructions ne comprenant pas la protéine FXR entière, mais seulement les domaines fonctionnels de FXR, le domaine de liaison du ligand suffit à lui seul pour l'action de la gugglesterone; (2) la drogue empêche l'interaction directe entre FXR et son co-activateur $S R C l$, induite par le ligand, ce qui a été montré en utilisant la technique du FRET (fluorescence energy transfer) ; (3) finalement, le lien entre le mode d'action moléculaire et l'effet hypocholestérolémiant est établi dans des expériences in vivo où la gugglesterone, administrée à des souris soumises à un régime enrichi en cholestérol, normalise l'accumulation de cholestérol hépatique. Cependant, la gugglesterone perd cette activité lorsqu'elle est administrée à des souris dont les deux copies du gène FXR ont été invalidées, ce qui démontre que ce facteur de transcription doit être présent pour que la gugglesterone puisse agir.

Cette étude est intéressante à plus d'un titre: d'un point de vue moléculaire, les antagonistes des facteurs de transcription de la famille des récepteurs nucléaires ne sont pas si nombreux dans l'arsenal pharmacologique, et il est en général plus facile d'activer que d'inhiber de tels facteurs. D'un point de vue clinique, cette étude démontre l'importance de la voie contrôlée par FRX dans la régulation globale de l'homéostasie du cholestérol, et fait de ce facteur une évidente cible thérapeutique. Du point de vue de sa structure chimique, la gugglesterone se compose d'un noyau stérol modifié, et s'apparente donc à la famille très vaste des stérols végétaux, regroupés sous le terme général de phytostérols. Certains de ces phytostérols ont la propriété de diminuer le taux de cholestérol circulant comme l'ont montré plusieurs études épidémiologiques, et sont même, depuis peu, utilisés comme additifs alimentaires dans des margarines. On peut donc se demander dans quelle mesure le mode d'action de la gugglesterone décrypté par les chercheurs signant l'article de Science, ne pourrait pas s'appliquer à cette gamme plus large de composés. Apparemment, les phytostérols seraient des analogues du cholestérol, qui ne seraient pas absorbés au niveau intestinal, mais le rôle de FXR dans cet aspect du métabolisme du cholestérol reste mal connu.

Enfin, il est toujours réconfortant d'apporter des explications modernes, par le biais de la science, à des pratiques mil-

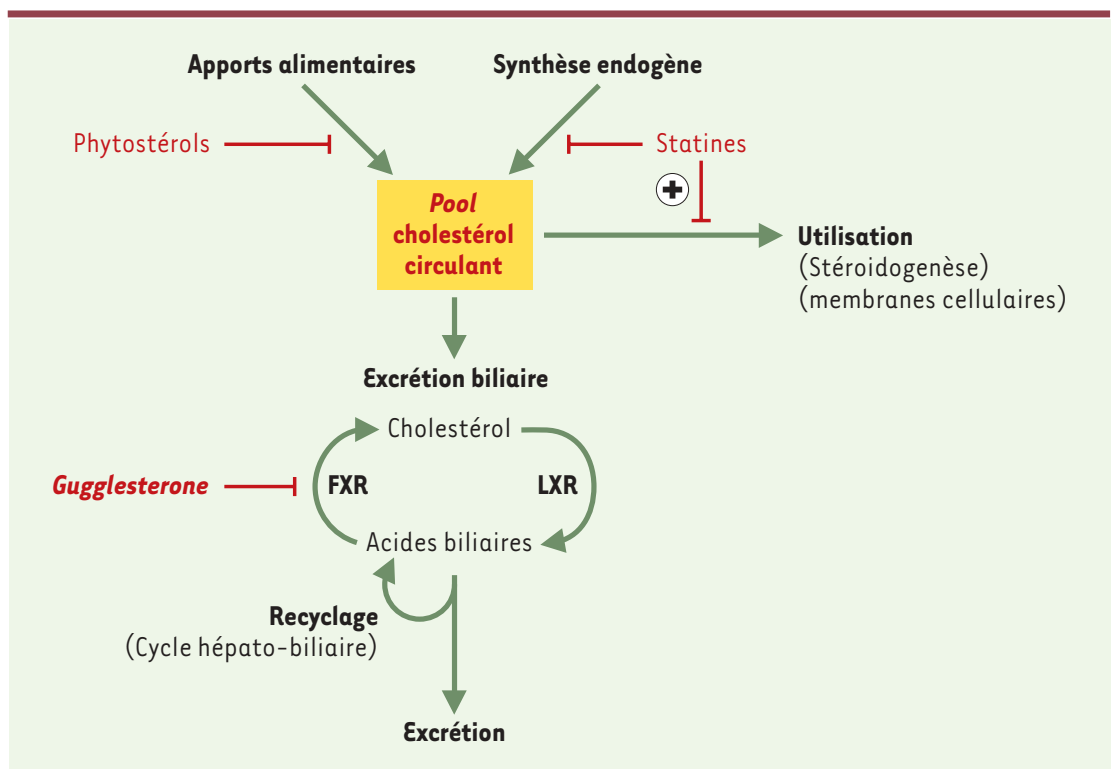

Figure 1. Représentation schématique des voies ciblées par les produits qui modulent la cholestérolémie. + indique une activation, $\longrightarrow$ un blocage. FXR : farnesoid $X$-activated receptor ; LXR : liver $X$ receptor. 
lénaires qui trouvent leur origine dans la médecine traditionnelle. II faut cependant se garder de confondre la guggle gum indienne, dont est extraite la gugglesterone hypocholestérolémiante, et la myrrhe offerte par les rois mages à l'enfant Jésus. Bien que très proches, les arbustes qui les produisent sont originaires de régions différentes, l'ćthiopie et l'Arabie pour la myrrhe (commiphora myrra), l'Inde pour la guggle gum (commiphora mukul). II se peut bien que ce produit précieux, recherché dans l'Antiquité pour toutes sortes de vertus, ne revienne à la mode dans notre monde moderne à cause de ses propriétés hypocholestérolémiantes! $\diamond$

Indian guggle gum to lower cholesterol

\section{NOUVELLE}

\section{Le couple CbI-CIN85 essentiel à l'endocytose des récepteurs}

Philippe Soubeyran récepteurs sont rapidement ubiquitinylés, internalisés par un mécanisme d'endocytose contrôlé par les proLudwig Institute for Cancer Research, Box 595, 75124, Uppsala, Suède. téines « clathrine » qui s'organisent en forme de cage autour de la vésicule

en formation, facilitant

> L'activation d'un récepteur par la liaison de son ligand soluble à la surface de la cellule peut déclencher son auto-phosphorylation. Survient ensuite le recrutement de diverses molécules accessoires sur la partie cytoplasmique du récepteur, et leur activation, déclenchant celle de multiples voies de signalisation intracellulaires, dont la plupart se caractérisent par des cascades de phosphorylation-activation des protéines impliquées. Les réponses engendrées sont variées, et affectent de nombreuses fonctions physiologiques de la cellule comme la prolifération, la migration, ou la différenciation cellulaire.

La plupart des voies de signalisation sont dotées d'un mécanisme de rétrocontrôle négatif qui limite la durée du signal et prévient une stimulation excessive de la cellule. La perturbation de cet équilibre peut engendrer des situations pathologiques dont le développement tumoral. L'une des façons d'arrêter le signal consiste à retirer physiquement le récepteur activé de la surface cellulaire, par internalisation. En effet, à la suite de leur activation, les

ainsi son invagination, et dégradés par un processus impliquant à la fois le protéasome et le lysosome [1]. Notre équipe [2], et celle de S. Giordano [3], ont éclairci le mécanisme d'internalisation de deux types de récepteurs, celui de l'EGF (epidermal growth factor) et celui de l'HGF (hepatocyte growth factor), en identifiant un partenaire essentiel, CIN85. C'est un couple, fait de Cbl et CIN85, qui fait le lien entre les récepteurs activés et les protéines endophiline qui contrôlent l'endocytose dépendante de la clathrine. Cbl est une protéine adaptatrice se liant à de nombreuses molécules de signalisation (Figure 1), membre d'une famille plus large qui contient d'autres protéines, $\mathrm{Cbl}-\mathrm{b}$ et Cbl-3 [4]. Cbl est dotée, à son extrémité $\mathrm{N}$-terminale, d'un domaine de liaison aux kinases activées PTB (phosphotyrosine tyrosine binding), et d'un domaine ring finger, qui catalyse le transfert de molécules d'ubiquitine de l'enzyme de conjugaison de l'ubiquitine vers la molécule cible (la kinase). L'extrémité C-terminale de $\mathrm{Cbl}$ comprend plusieurs domaines d'interaction moléculaire avec de nombreuses protéines de signalisation : (1) un

Figure 1. Représentation schématique du rôle de Cbl, CIN85, et des endophilines dans l'internalisation des récepteurs. Le mode d'interaction des trois protéines conduisant à la formation du complexe trimérique responsable de l'internalisation des récepteurs activés est illustré. Le domaine PTB (phosphotyrosine binding domain) se lie aux récepteurs activés. Le domaine ring finger (RF) confère l'activité de ligase de l'ubiquitine; Pro-Rich : région riche en résidus proline; Ac-Box (acidic box) contient plusieurs résidus tyrosine phosphorylables; LZ (leucine zipper): domaine impliqué dans la dimérisation de $\mathrm{Cbl}$; $\mathrm{SH} 3$ (Src homology 3 ) : domaine qui se lie à des motifs proline PxxP ; Cc (coiled-coil) : domaine impliqué dans l'oligomérisation de CIN85; LPAAT : activité acide lysophosphatidique acyltransférase des endophilines. 